\title{
Reconstruction method for fluorescence molecular tomography based on L1-norm primal accelerated proximal gradient
}

\author{
Yuhao Liu \\ Shixin Jiang \\ Jie Liu \\ Yu An \\ Guanglei Zhang \\ Yuan Gao \\ Kun Wang \\ Jie Tian
}




\title{
Reconstruction method for fluorescence molecular tomography based on L1-norm primal accelerated proximal gradient
}

\author{
Yuhao Liu, ${ }^{a, b, \dagger}$ Shixin Jiang, ${ }^{a, b, \dagger}$ Jie Liu, ${ }^{a, *}$ Yu An, ${ }^{b}$ Guanglei Zhang, ${ }^{c}$ Yuan Gao, ${ }^{b}$ Kun Wang, ${ }^{b, d, *}$ and Jie Tian ${ }^{b, d, e, *}$ \\ ${ }^{a}$ Beijing Jiaotong University, School of Computer and Information Technology, Haidian District, Beijing, China \\ Institute of Automation, CAS Key Laboratory of Molecular Imaging, Chinese Academy of Sciences, Beijing, China \\ ${ }^{\circ}$ Beihang University, School of Biological Science and Medical Engineering, Beijing Advanced Innovation Center for Biomedical Engineering, \\ Beijing, China \\ dUniversity of Chinese Academy of Sciences, Beijing, China \\ eBeijing Key Laboratory of Molecular Imaging, Beijing, China
}

\begin{abstract}
Fluorescence molecular tomography (FMT) has been widely used in preclinical tumor imaging, which enables three-dimensional imaging of the distribution of fluorescent probes in small animal bodies via image reconstruction method. However, the reconstruction results are usually unsatisfactory in the term of robustness and efficiency because of the ill-posed and ill-conditioned of FMT problem. In this study, an FMT reconstruction method based on primal accelerated proximal gradient (PAPG) descent and L1-norm regularized projection (L1RP) is proposed. The proposed method utilizes the current and previous iterations to obtain a search point at each iteration. To achieve fast convergence, the PAPG method is applied to efficiently solve the search point, and then L1RP is performed to obtain the robust and accurate reconstruction. To verify the performance of the proposed method, simulation experiments are conducted. The comparative results revealed that it held advantages of robustness, accuracy, and efficiency in FMT reconstructions. Furthermore, a phantom experiment and an in vivo mouse experiment were also performed, which proved the potential and feasibility of the proposed method for practical applications. ๑ 2018 Society of Photo-Optical Instrumentation Engineers (SPIE) [DOI: 10.1117/1.JBO.23.8.085002]
\end{abstract}

Keywords: fluorescence molecular tomography; search point; primal accelerated proximal gradient; L1-norm regularized projection; image reconstruction.

Paper 180090RR received Feb. 20, 2018; accepted for publication Jul. 30, 2018; published online Aug. 14, 2018.

\section{Introduction}

Fluorescence molecular tomography (FMT) is an emerging imaging modality that enables three-dimensionally (3-D) quantitative observation of imaging targets and pathways at the molecular and cellular level. ${ }^{1-6}$ FMT has been widely used in the preclinical research of oncology, which can noninvasively show the dynamic interactions of fluorescent targets. Because of its quantification characteristics, FMT has been considered as an important tool for tumor diagnostic imaging and basic researches. ${ }^{7-10}$

The process of FMT usually includes the forward problem and the inverse problem. The FMT reconstruction is a typical inverse problem based on the system matrix and measurement data sets that obtained by the forward problem. However, it is very challenging to solve the FMT inverse problem efficiently and precisely. ${ }^{11}$ FMT reconstruction is an ill-posed problem due to the multiple scatterings of photons when propagating through heterogeneous biological tissues. Besides, since only the photon distribution on the surface is measurable, the FMT reconstruction is always ill-conditioned. ${ }^{12-15}$ Furthermore, since FMT reconstruction is sensitive to noise, it is difficult to obtain satisfactory results under the influence of the system noise, such as autofluorescence and the shot noise of the charge-coupled

*Address all correspondence to: Jie Liu, E-mail: jieliu@ bjtu.edu.cn; Kun Wang, E-mail: kun.wang@ia.ac.cn; Jie Tian, E-mail: jie.tian@ia.ac.cn

†These authors contributed equally to this work. device (CCD) camera. ${ }^{16}$ Therefore, how to precisely and efficiently solve the inverse problem is important for FMT study.

Over past decades, great efforts were made to develop various reconstruction methods. The regularization methods are widely used in the inverse problem to mitigate the ill-posedness. Among them, the L2-norm regularization is commonly used, and the primary benefit of using L2-norm regularization is the simplicity of the optimization problem involved, which can be efficiently solved by standard minimization tools, such as Newton's method and conjugate gradient method (CG_L2). ${ }^{17-20}$ However, the performance of L2-norm is usually getting worse while existing high noise, and the reconstruction of L2-norm is likely to be oversmoothed. To overcome the oversmoothness of L2-norm regularization method, a priori information, which is sparsity, is adopted in FMT. For FMT, the size of early-stage tumors is small and sparse compared with the whole body of the imaging object, so the L1-norm regularization was employed to reconstruct the fluorescent source. According to the compressed sensing theory, ${ }^{21}$ many solution schemes combined with L1-norm optimization algorithm have been exploited to solve this problem, such as the iterated shrinkage method (IS_L1), L1-norm regularization piecewise constant level set approach (L1-PCLS), variable splitting and alternating direction (VSAD) scheme, nonconvex regularization method, and the stagewise orthogonal matching pursuit-based method (StOMP). ${ }^{22-26}$ These methods have been proved superior in

$1083-3668 / 2018 / \$ 25.00$ @ 2018 SPIE 
overcoming the oversmoothing limitation of the L2-norm regularization. ${ }^{27-29}$ However, measurement noises are unavoidable in FMT experiments. The methods mentioned above are not robust enough in presence of measurement noise.

In this study, we present a robust and efficient reconstruction method based on the L1-norm primal accelerated proximal gradient (L1-PAPG) for FMT reconstruction. The PAPG method has been proposed in multitask learning in previous studies. ${ }^{30}$ Here, we adopted it to accelerate the computational process during the iterative process. At each iteration, the value of the next iteration always relied on a search point that combined its previous and current iterations. This strategy was designed to mitigate the noise jamming and obtain more precise and robust results. Besides, in order to utilize the sparsity of fluorescent sources, the L1-norm regularized projection (L1RP) was employed to achieve L1-norm constraint. In this process, we introduced the Lipschitz constant to initialize the step size. ${ }^{31}$ To validate the performance of the L1-PAPG method for FMT reconstruction, simulation experiments were conducted. We compare the proposed method with two recent reported reconstruction methods, the VSAD method based on L1-norm and the $1_{2,1}$-norm optimization $\left(\mathrm{L}_{2,1}\right.$-norm) method based on structured sparsity. The results showed that L1-PAPG achieved more robust and accurate reconstructions than VSAD and obtained similar results with $\mathrm{L}_{2,1}$-norm method with faster speed. Moreover, the mouse phantom experiment and the in vivo small mouse experiment also proved that the proposed method had great potential for its application in tumor mouse model imaging.

The contents of this manuscript are as follows. In Sec. 2, we present the photon propagation model and provide the proposed method of FMT reconstruction. In Sec. 3, the simulation experiments, mouse phantom experiments, and the in vivo experiments were conducted to verify the peformance of the L1-PAPG method. Finally, we summarize the paper and make a conclusion in Sec. 4.

\section{Method}

\subsection{Model of Photon Propagation}

For the steady-state FMT with point excitation light sources, the photon propagation can be formulized by coupled diffusion equation. ${ }^{32,33}$ To solve the coupled equation, the Robin-type boundary conditions are introduced. ${ }^{34}$ Then, based on the finite-element theory, the FMT problem can be linearized and obtain the following matrix-form equations:

$b=A X$

where the measurement dataset of FMT is marked with the symbol $b$, and the weight matrix FMT system is marked with alphabet $A$. The intensity matrix of the fluorescence distribution in biological tissues is marked with the alphabet $X{ }^{22}$ The inverse problem of FMT is to solve the intensity matrix $X$ in the linear math Eq. (1). More detailed description can be found in Ref. 15 .

\subsection{Method Based on Primal Accelerated Gradient Descent and L1-Norm Regularized Projection (L1-PAPG) for Reconstruction}

Because of the sparsity of the fluorescent sources, the L1-norm regularization is employed in FMT problem to get the sparsity of the solution. ${ }^{35-37}$ Hence, Eq. (1) can be transformed into the following optimization function:

$\min _{x} E(x)=\frac{1}{2}\|A X-b\|_{2}^{2}+\lambda\|X\|_{1} \quad$ subject to $x \geq 0$,

where $g(x)=\|X\|_{1}$ is the L1-norm of matrix $X$, where $f(x)=$ $\frac{1}{2}\|A X-b\|_{2}^{2}$ denotes the objective function, the regularization constraint parameter $\lambda$ is used to balance $f(x)$ and $g(x)$.

The conventional L1-norm regularization method cannot handle the high-noise condition. ${ }^{14,29}$ The results usually are imprecise and have a lot of artifacts. In this work, we proposed a robust and efficient method based on PAPG and L1RP, ${ }^{30,31}$ which can effectively find the optimal solution.

The PAPG method based on two matrices $s$ and $x$, where $s$ is the search point and $x$ is the approximate solution. The search point $s$ can be obtained as follows:

$s_{i}=x_{i}+\alpha_{i}\left(x_{i}-x_{i-1}\right)$,

where $\alpha_{i}$ is the combination coefficient associated with variable $t$ :

$\alpha_{i}=\frac{t_{i-1}}{t_{i-2}}-t_{i-1}$

to accelerate the convergence, $t_{i}$ should decrease and tend to zero as fast as possible. $t_{i}$ is calculated as follows:

$t_{i}=\frac{\sqrt{t_{i-1}^{4}+4 t_{i-1}^{2}}-t_{i-1}^{2}}{2}$.

We first calculated the antigradient of $s$ to get $v$ :

$v_{i}=s_{i}-\frac{1}{\gamma_{i}} \nabla f\left(s_{i}\right)$,

where $\frac{1}{\gamma_{i}}$ is the step size, and the Armijo-Goldstein rule is used to linearly search $\gamma_{i}$. To make more stable, we utilize the Lipschitz condition to get the initial value of $\gamma_{i}$. In this case, both $f(x)$ and $g(x)$ are convex functions, and the function $f(x)$ satisfies the Lipschitz condition:

$\left\|\nabla f(x)-\nabla f\left(x^{\prime}\right)\right\| \leq L\left\|x-x^{\prime}\right\| \quad \forall x, \quad x^{\prime} \in X$,

where $L$ is the Lipschitz constant: $L=\lambda_{\max }\left(A^{T} A\right), \lambda_{\max }$ means the maximal eigenvalue of $A^{T} A$, we initialize $\gamma_{i}$ with the Lipschitz constant. ${ }^{30}$

The approximate solution $x_{i+1}$ is computed as the L1RP, which is obtained by the Euclidean projection of $v$ onto convex set $G:^{31}$

$x_{i+1}=\min _{x \in G} \frac{1}{2}\left\|x_{i}-v_{i}\right\|^{2}$.

We consider that the $x$ reaches the optimal approximate solution if the following equation holds:

$\|d\|_{2}^{2} \leq \lambda \quad$ or $\quad\|A d\|_{2}^{2} \leq \gamma\|d\|_{2}^{2}$,

where $d=x_{i+1}-s_{i}$ is the difference between the new approximate solution $x$ and the search point $s$. The illustration of the proposed method is shown in Fig. 1. The L1-PAPG method mainly contains two steps, first, utilizing the PAPG method 


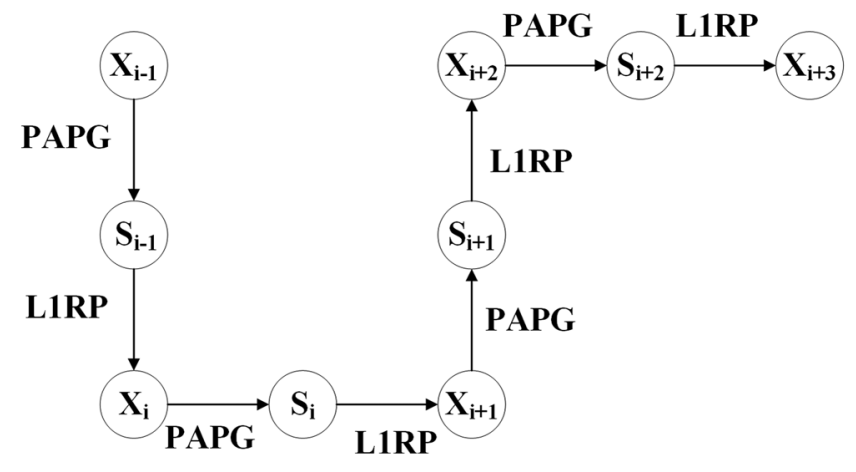

Fig. 1 The illustration of the L1-PAPG method.

to find the search point, and then using L1RP method to obtain the approximate solution.

In FMT, the reconstruction results are sensitive to the regularization parameter. Thus, the selection of the regularization parameter is important for FMT problem. In this work, to obtain the optimal solution and make the results more reliable, the L-curve criterion was adopted to determine the regularization parameters of all methods. The L-curve criterion is based on a $\log -\log$ plot of corresponding values of residual and solution norms, which is $\left(\log \|A x-b\|_{2}, \log \|x\|_{1}\right)$. The optimal regularization parameter is determined by the point with maximum curvature of the L-shaped region. ${ }^{38}$ The L-curve method was widely used in adaptive parameter selection of FMT study and was proofed that it is an effective and reliable method for FMT problem. ${ }^{39,40}$ In this study, all the methods with L-curve parameters were implemented by using MATLAB regularization toolbox. ${ }^{41}$

The flowchart of the main steps of L1-PAPG method is given in Algorithm 1.

\section{Results}

In this section, we conducted the heterogeneous simulation experiments, mouse phantom experiments, and in vivo small mouse experiments for evaluating the performance of the L1PAPG method. All reconstruction programs were conducted by MATLAB and ran on a desktop computer with 16 GB RAM and 3.40 GHz Intel Core i7-6700 CPU.

For quantitative analysis, the signal-to-background ration (SBR) and the position error (PE) are introduced in the paper. SBR is adopted to demonstrate the contrast of the reconstruction source and background, which is defined as follows:

$\mathrm{SBR}=\frac{\mu_{\mathrm{ROI}}-\mu_{\mathrm{ROB}}}{\sqrt{\omega_{\mathrm{ROI}} \sigma_{\mathrm{ROI}}^{2}+\omega_{\mathrm{ROB}} \sigma_{\mathrm{ROB}}^{2}}}$,

where $\mu$ is the mean value, $\sigma$ is the standard deviation, and $\omega$ is the weight coefficient. The subscripts ROI and ROB mean the region of interest (ROI) and region of background (ROB), respectively.

PE aims to calculate the barycenter deviation between the real fluorescent region and the reconstruction region, which is given by

$$
\begin{aligned}
\mathrm{PE} & =\left\|P_{r}-P_{0}\right\|_{2} \\
& =\sqrt{\left[P_{r}(x)-P_{0}(x)\right]^{2}+\left[P_{r}(y)-P_{0}(y)\right]^{2}+\left[P_{r}(z)-P_{0}(z)\right]^{2}},
\end{aligned}
$$

Algorithm 1 The L1-PAPG method.

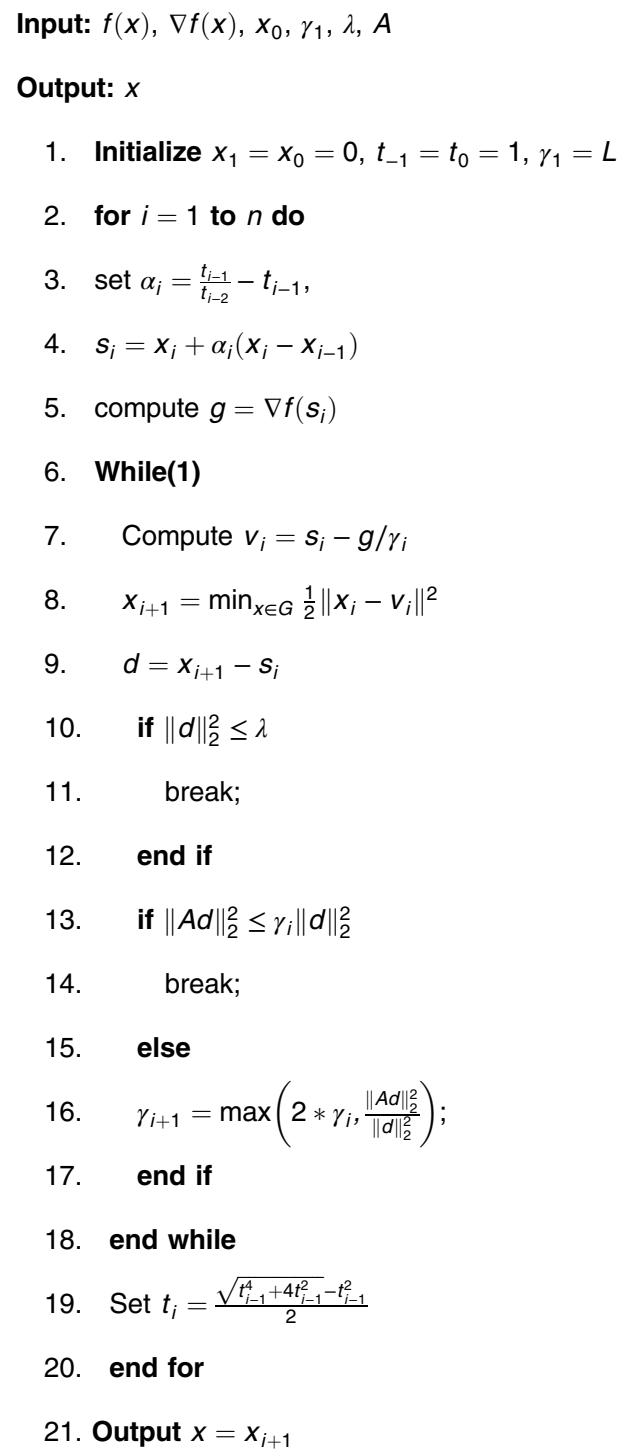

where $P_{r}$ is the geometric central position of the reconstruction fluorescent source and $P_{0}$ is the actual position of the fluorescent source, and $(x, y, z)$ is the $3-\mathrm{D}$ coordinate of the centroid.

\subsection{Heterogeneous Simulation Model Experiment}

A heterogeneous simulation model was designed to evaluate the performance of the L1-PAPG method. The simulation model is $2 \mathrm{~cm}$ in height and $2 \mathrm{~cm}$ in diameter, as shown in Fig. 2. The simulated lungs, heart, bone, and muscle were represented by four kinds of simulated materials, whose optical parameters are presented in Table 1. The corresponding excitation and emission light wavelength are 780 and $830 \mathrm{~nm}$, respectively. ${ }^{42}$ In Fig. 2(b), the red dots marked different positions of the excitation light source. And two globular fluorescent sources, $\mathrm{S} 1$ and $\mathrm{S} 2$, were placed in the right lung. The fluorescent yield of each source was $0.02 \mathrm{~mm}^{-1}$. Both sources were $2 \mathrm{~mm}$ in diameter, and the center of the source was situated in $z=0$ plane. We measured the emitted fluorescent within a 160-deg field of view (FOV) at the opposite side of each 


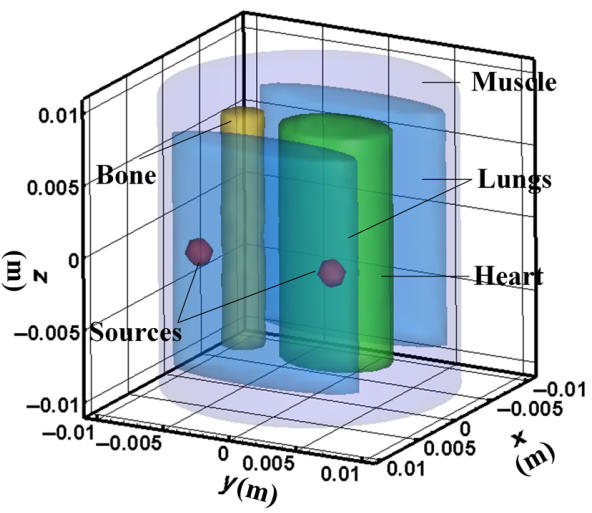

(a)

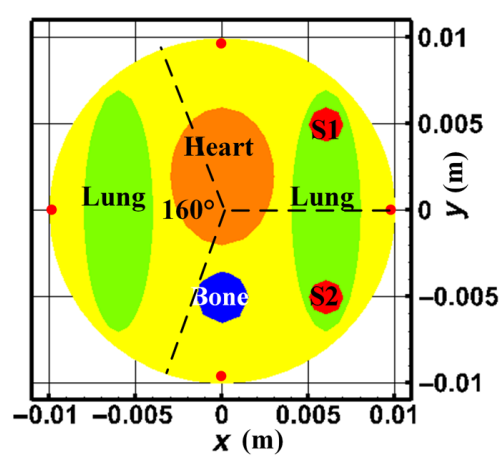

(b)

Fig. 2 The two-source heterogeneous simulation model. (a) The 3-D view of the model. (b) The crosssection in the $z=0$ plane. The red dots in panel (b) denote the excitation point sources. Fluorescence was collected from the opposite cylindrical surface within 160-deg FoV for each point source.

Table 1 Optical parameters of the heterogeneous model.

\begin{tabular}{lcccc} 
Material & $\left.\mu_{\mathrm{ax}} \mathrm{m}^{-1}\right)$ & $\mu_{\mathrm{sx}}^{\prime}\left(\mathrm{m}^{-1}\right)$ & $\mu_{\mathrm{am}} \cdot\left(\mathrm{m}^{-1}\right)$ & $\mu_{\mathrm{sm}}^{\prime}\left(\mathrm{m}^{-1}\right)$ \\
\hline Muscle & 38.9 & 279.4 & 29.6 & 234.5 \\
Lungs & 84.2 & 2005.6 & 61.7 & 1940.6 \\
Heart & 27.6 & 775.5 & 22.0 & 709.6 \\
Bone & 26.7 & 1995.5 & 20.1 & 1821.3 \\
\hline
\end{tabular}

Table 2 Quantitative analysis of different methods.

\begin{tabular}{lccc}
\hline Method & VSAD & $\mathrm{L}_{2,1}$-norm & L1-PAPG \\
\hline PE $(\mathrm{mm})$ & $\mathrm{S} 1: 0.24$ & $\mathrm{~S} 1: 0.18$ & $\mathrm{~S} 1: 0.18$ \\
& $\mathrm{~S} 2: 0.44$ & $\mathrm{~S} 2: 0.27$ & $\mathrm{~S} 2: 0.30$ \\
SBR & 22.1 & 76.86 & 67.37 \\
Time (s) & 14.33 & 7.12 & 6.64 \\
\hline
\end{tabular}
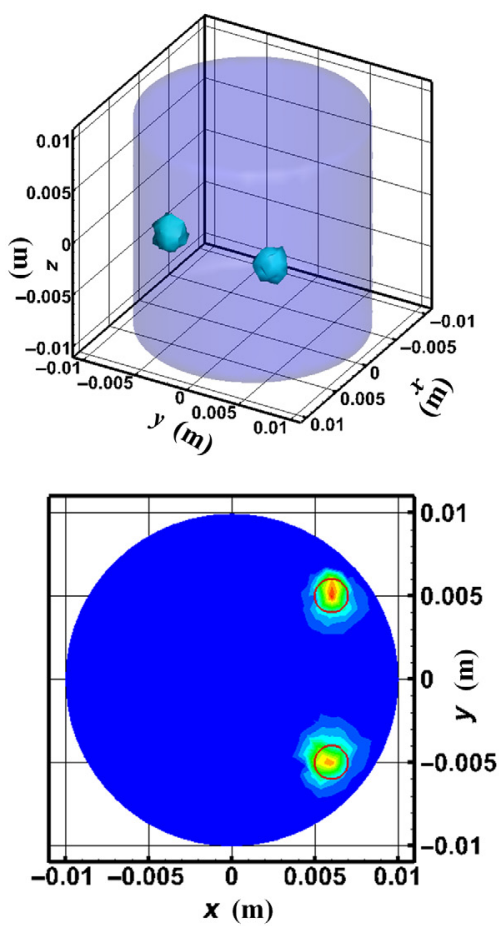

(a)
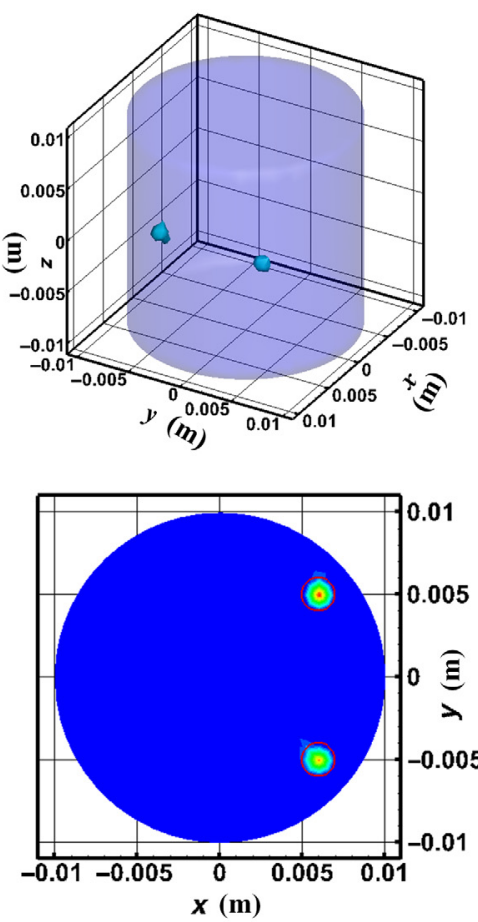

(b)
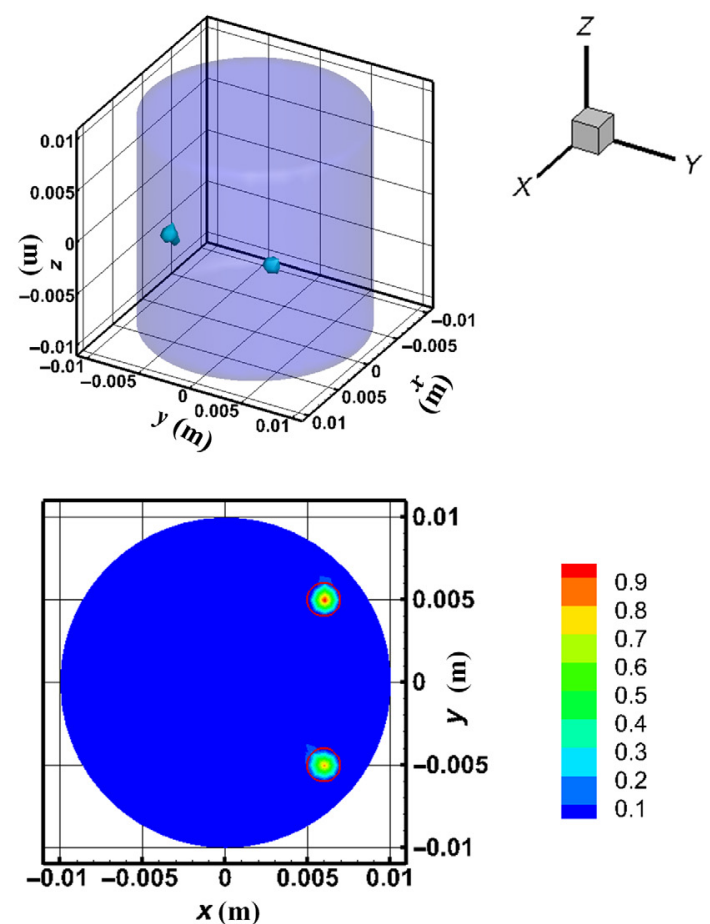

(c)

Fig. 3 Reconstruction results from the (a) VSAD method, (b) the $L_{2,1}$-norm method, and (c) the L1-PAPG method. The first row illustrates the 3-D views of the reconstruction results and the second row illustrates the slice images in the $z=0$ plane. The red circles in the slice images represent the real locations of the fluorescent sources. 
Noise

intensity

VSAD_3D

L2,1-norm_3D

L2,1-norm_CV

L1-PAPG 3D
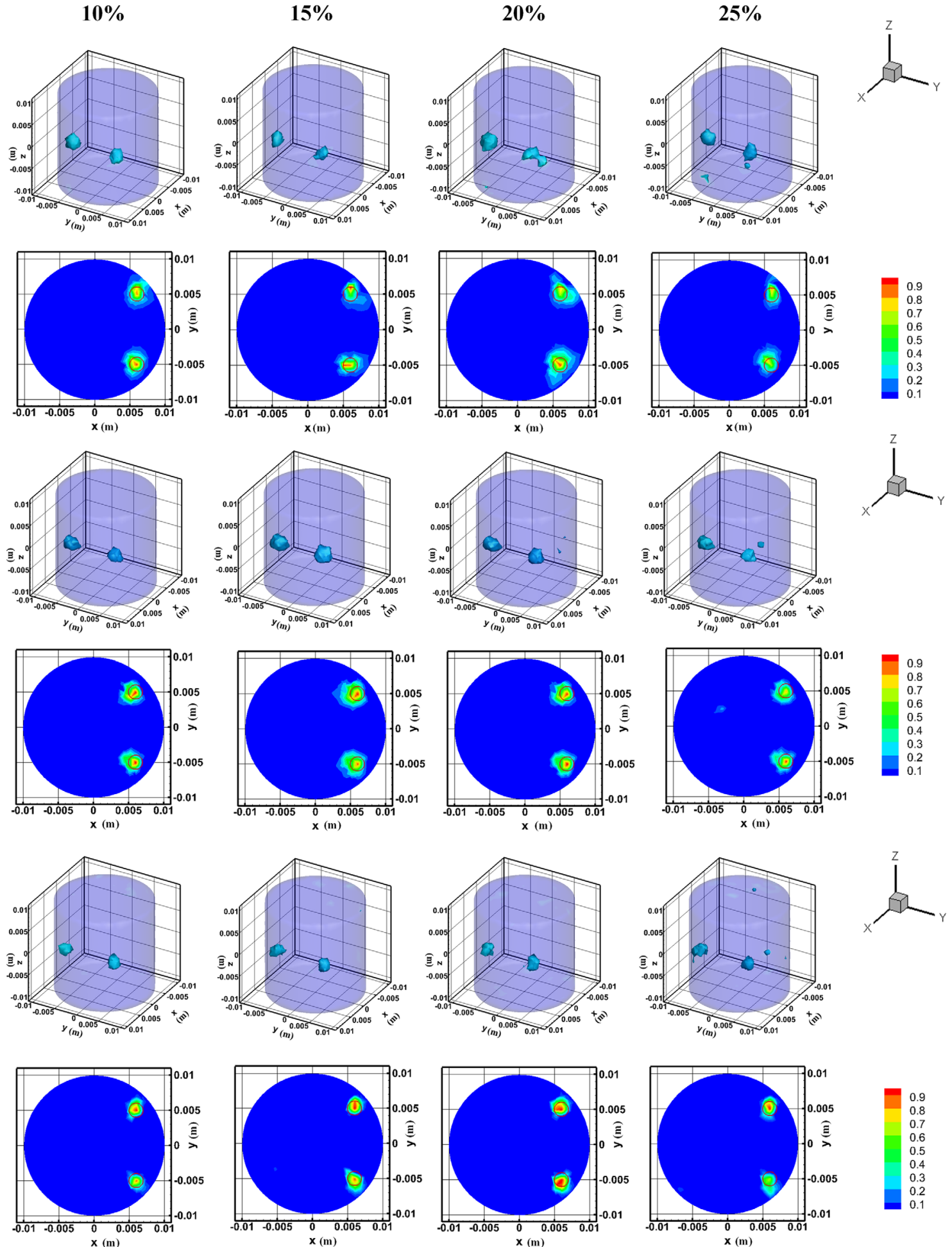

Fig. 4 Reconstructed results with different noise intensities for three methods. The columns denote different noise intensities $(10 \%, 15 \%, 20 \%$, and $25 \%$ ) corresponding to different reconstructed results with three methods. 3D denotes the 3-D views of the reconstructed results, and CCV denotes the cross-sectional views. The first and second rows are the reconstructed results obtained by the VSAD. The third and fourth rows correspond to the $L_{2,1}$-norm method. The last two rows correspond to the L1-PAPG method. The red circles in the cross-sectional views show the real positions of the fluorescent sources.

excitation light source through the simulation model. In the process of reconstruction, this heterogeneous simulation model with two sources was discretized into 5623 nodes and 33,490 tetrahedral elements.
To further verify the performance of L1-PAPG method, two other reconstruction methods, the VSAD and $\mathrm{L}_{2,1}$-norm method, were implemented to compare with the proposed method. The VSAD method used variable splitting strategy as well as 
alternating direction strategy for FMT reconstruction, which was accurate and efficient for FMT imaging. ${ }^{25}$ The $\mathrm{L}_{2,1}$-norm method utilized the group sparsity of the fluorescent source and adopted Nesterov's method to accelerate the computation, which can enhance robustness to noise. ${ }^{14}$ To make the results stable and convincing, the regularization parameters of all the methods were obtained by L-curve method, and the iterative number was set to 400 for all methods to ensure the convergence. The reconstruction results of three methods are shown in Fig. 3, and the quantitative analysis is shown in Table 2. Compared with the VSAD method, the L1-PAPG method can achieve a much smaller PE value and higher SBR. However, since the $\mathrm{L}_{2,1}$-norm included additional information, which was structured sparsity, the accuracy of the proposed method was not as good as $\mathrm{L}_{2,1}$-norm, but the PE gap between the two methods was small. Besides, since the proposed method only utilizes the sparsity information, the computational complex is less than $\mathrm{L}_{2,1}$-norm method. Thus, its reconstruction speed is faster. The phantom and in vivo experiment in next section also verified the conclusion.

As mentioned in Sec. 1, the noise in FMT is unavoidable. Thus, the robustness of reconstruction method is important for FMT reconstruction. In this experiment, we tested the robustness of the proposed method with noise corrupted data. The measurement datasets were artificially interfered by $10 \%, 15 \%, 20 \%$, and $25 \%$ Gaussian noise, respectively. The reconstruction images of three methods under different noise intensities are demonstrated in Fig. 4. The quantitative analysis of the reconstruction results is summarized in Table 3. It is clear that when the noise intensity increased, the L1-PAPG method and $L_{2,1}$-norm method offered more robust reconstruction of two fluorescent sources compared with the VSAD methods. Even if the measurement dataset was artificially interfered

Table 3 Quantitative analysis of the three methods with different noise intensities.

\begin{tabular}{|c|c|c|c|c|c|}
\hline \multirow[b]{2}{*}{ Method } & \multirow[b]{2}{*}{ Noise intensities } & \multicolumn{2}{|c|}{$\mathrm{PE}(\mathrm{mm})$} & \multirow[b]{2}{*}{ SBR } & \multirow[b]{2}{*}{ Time (s) } \\
\hline & & S1 & S2 & & \\
\hline VSAD & $10 \%$ & 0.34 & 0.48 & 21.75 & 14.35 \\
\hline $\mathrm{L}_{2,1}$-norm & & 0.22 & 0.31 & 67.76 & 7.33 \\
\hline L1-PAPG & & 0.27 & 0.35 & 60.97 & 6.65 \\
\hline VSAD & $15 \%$ & 0.47 & 0.56 & 19.72 & 14.37 \\
\hline $\mathrm{L}_{2,1}$-norm & & 0.27 & 0.38 & 55.21 & 7.41 \\
\hline L1-PAPG & & 0.32 & 0.42 & 53.6 & 6.85 \\
\hline VSAD & $20 \%$ & 0.53 & 0.56 & 16.27 & 14.42 \\
\hline $\mathrm{L}_{2,1}$-norm & & 0.44 & 0.45 & 44.15 & 7.31 \\
\hline L1-PAPG & & 0.44 & 0.47 & 43.75 & 6.67 \\
\hline VSAD & $25 \%$ & 0.58 & 0.90 & 16.20 & 14.39 \\
\hline $\mathrm{L}_{2,1}$-norm & & 0.47 & 0.55 & 35.51 & 7.24 \\
\hline L1-PAPG & & 0.45 & 0.57 & 34.97 & 6.54 \\
\hline
\end{tabular}

by $25 \%$ Gaussian noise, the proposed method still achieved satisfactory results. Table 3 also demonstrates that, for the same noise intensity, the L1-PAPG method offered the similar performance with $\mathrm{L}_{2,1}$-norm, and with the noise intensity increased, the L1-PAPG method was robust and less effected by the noise. It is further proved that the L1-PAPG method can obtain the better results than traditional methods and can obtain similar results with $\mathrm{L}_{2,1}$-norm with faster reconstruction speed.

\subsection{Mouse Phantom Experiments}

In this experiment, a dual-modality imaging system equipped with micro-CT and FMT, which was established by our team, was used for data acquisition, ${ }^{43-45}$ as shown in Fig. 5. The system mainly consisted of a rotating stage, a micro-CT with $\mathrm{x}$-ray generator (UltraBright, Oxford Instruments, USA) and $\mathrm{X}$-ray detector (CMOS Flat-panel Detector, Hamamatsu, Japan), an ultrasensitive cooled CCD camera (PIXIS 1024BR, Princeton Instruments, USA), and a continuous wave laser (the center wavelength is $671 \mathrm{~nm}$ ).

To further verify the feasibility and performance of the L1-PAPG method, the mouse phantom experiment (XFM-2 Fluorescent Phantom, PerkinElmer, USA) was implemented, as shown in Fig. 6. The phantom includes the main body and two fluorescent tubes. On the top of tube 1 is fluorescent sources, the corresponding peak excitation wavelength and emission wavelength is 671 and $710 \mathrm{~nm}$, respectively, which is the same with cy5.5 probe. Tube 2 is blank, which is same with the main body, as shown in Fig. 6(a). The red box in Fig. 6(a) represents the ROI of reconstruction.

The main process of the mouse phantom experiments as follows. First, the optical data were acquired at a detector FOV of $160 \mathrm{deg}$ and four projections with different angles were adopted, and each angle was acquired once. Then, the phantom was scanned with micro-CT, as shown in Fig. 6(c). The red circle in Fig. 6(c) was the location of the fluorescent source (21.00 mm, $23.00 \mathrm{~mm}$, and $18.00 \mathrm{~mm}$ ). Next, to describe the photon distribution on the surface of the phantom, the fusion of the mesh and the fluorescence data were carried out via a 3-D surface flux reconstruction algorithm, ${ }^{46}$ as shown in Fig. 6(b). Finally, the mouse phantom was discretized into a volumetric mesh with 5693 nodes and 34,017 tetrahedral elements.

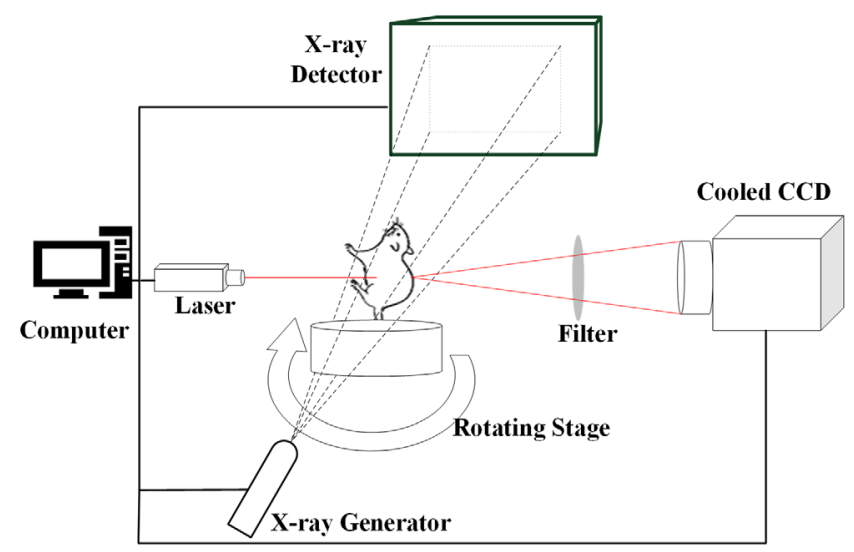

Fig. 5 The schematic illustration of the dual-modality micro-CT and FMT imaging system. 


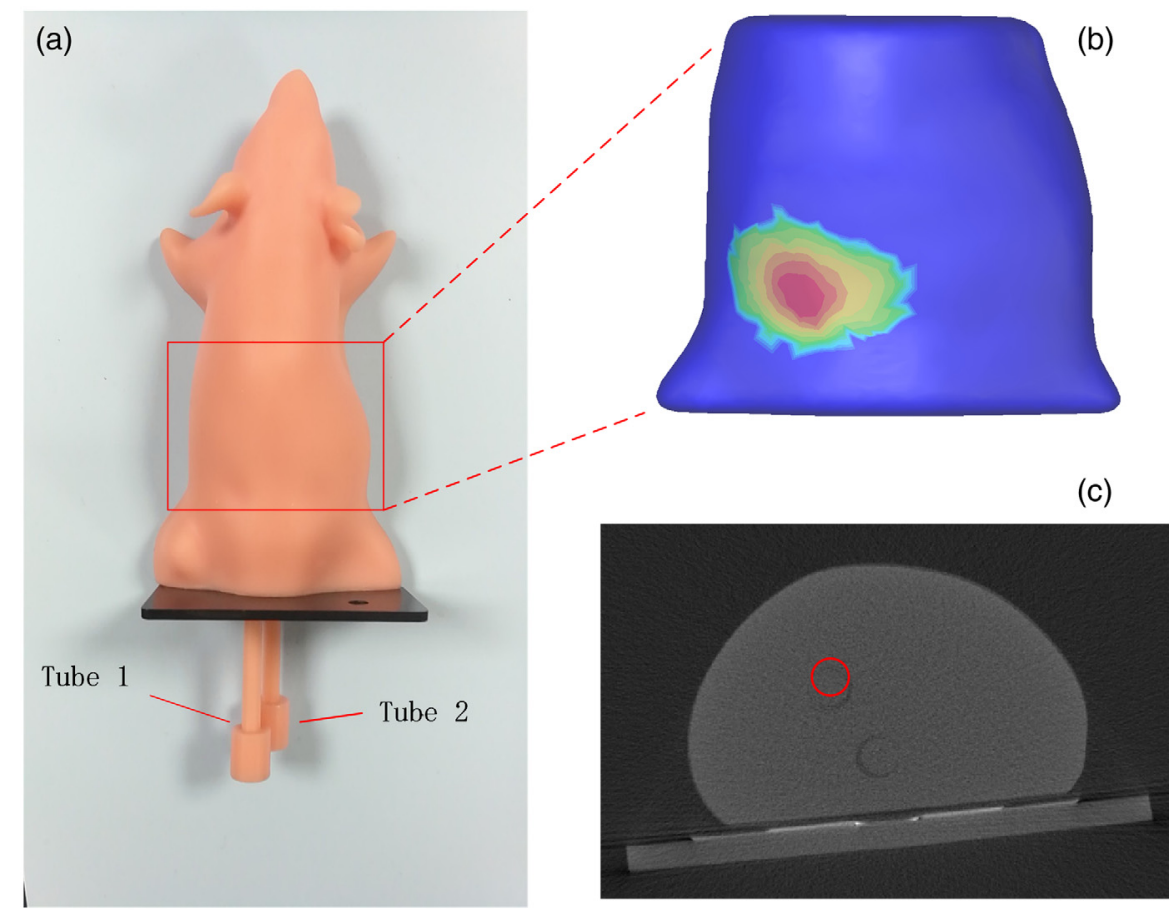

Fig. 6 (a) The illustration of mouse phantom. (b) Illustration of photon distribution on the surface. (c) The CT slice of $z=18 \mathrm{~mm}$ of the mouse phantom, the red circle in panel (c) represents the fluorescent source The illustration of the dual-modality micro-CT and FMT imaging system.
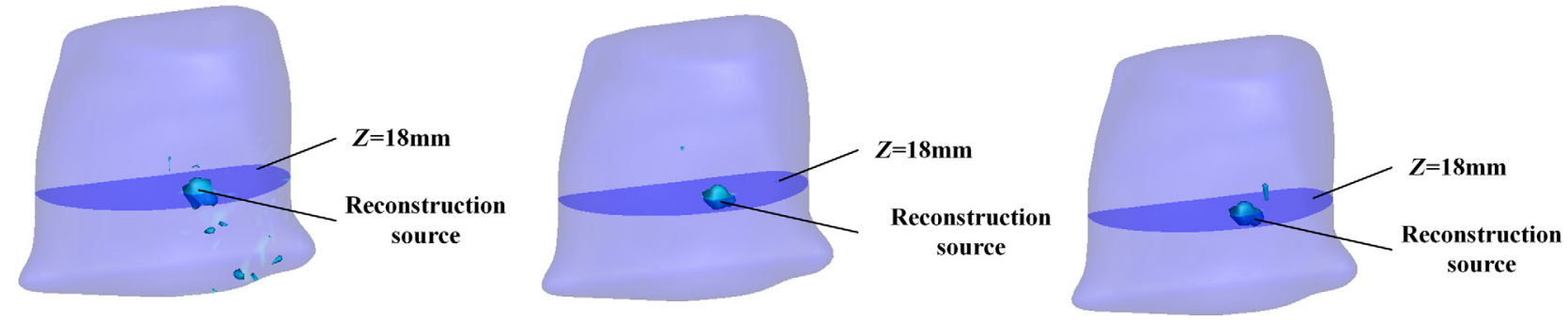

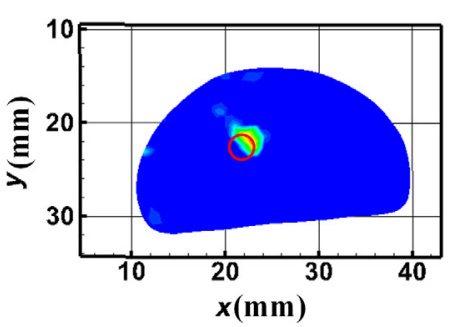

(a)

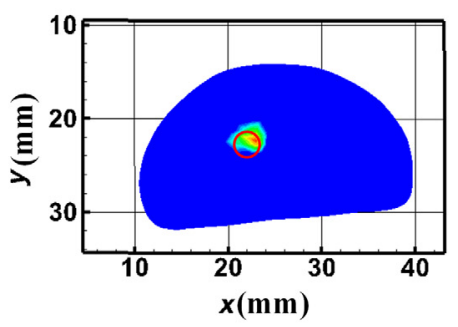

(b)

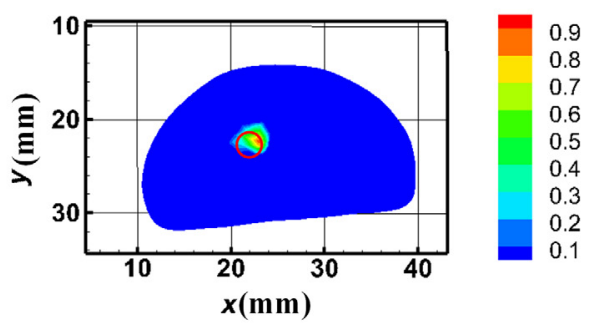

(c)

Fig. 7 The reconstruction results of the three methods. (a) The results of VSAD method, (b) results of the $L_{2,1}$-norm method, and (c) the results of L1-PAPG method. The red circle represents the location of the source.

Table 4 Quantitative analysis of the mouse phantom experiment.

\begin{tabular}{lccc} 
& VSAD & $\mathrm{L}_{2,1}$-norm & L1-PAPG \\
\hline PE $(\mathrm{mm})$ & 0.76 & 0.56 & 0.58 \\
SBR & 40.81 & 79.68 & 70.44 \\
Time (s) & 15.90 & 7.43 & 6.02 \\
\hline
\end{tabular}

After the above process, the L1-PAPG method was also compared with the VSAD and $\mathrm{L}_{2,1}$-norm method. Similarly, the parameters of the three methods were chosen by L-curve method. The reconstruction results of the three methods were shown in Fig. 7, and the quantitative analysis of the reconstruction results can be found in Table 4. From the results, the L1-PAPG could also obtain the better results in PEs and SBRs than VSAD, and obtain the similar results with $\mathrm{L}_{2,1}$-norm with faster speed, which further demonstrate the advantage of the proposed method. 


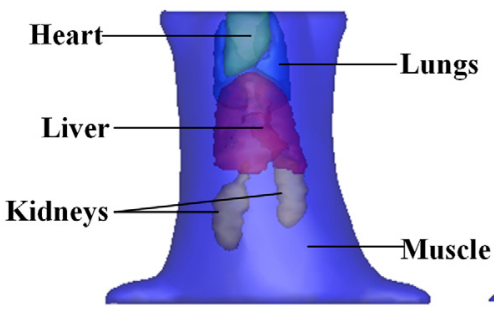

(a)

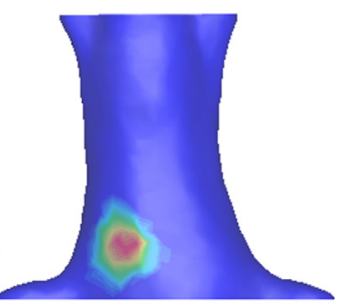

(b)
Fig. 8 (a) The heterogeneous mouse model for in vivo experiment and (b) photon distribution on the surface.

\subsection{In-Vivo Small Mouse Experiments}

To validate the potential of the practical application of the L1-PAPG method, an in vivo small mouse experiment was implemented. In this experiment, a fluorescent bead $(3 \mathrm{~mm}$ in diameter) containing cy5.5 solution was implanted into the hypogastria of the mouse. The extinction coefficient of the cy5.5 solution is $0.019 \mathrm{~mm}^{-1} \mu \mathrm{M}^{-1}$, and the quantum efficiency is 0.23 . The corresponding peak excitation wavelength and emission wavelength is 671 and $710 \mathrm{~nm}$, respectively. ${ }^{47}$ The data acquisition and procedure are as same as those in mouse phantom experiment. The Feldkamp-Davis-Kress method was utilized to structure the mouse stereoscopic data after scanning, ${ }^{48}$ as shown in Fig. 8(a). The major organs (heart, kidneys, muscle, lungs, and liver) of the mouse were segmented and marked with different colors, and the optical property parameters of these organs were listed in Table $5 .{ }^{49}$ And the photon distribution on the surface was shown in Fig. 8(b).

The micro-CT images were shown in Fig. 9. The green square marks the position of the fluorescent bead at the coordinates $(43.00 \mathrm{~mm}, 47.00 \mathrm{~mm}$, and $6.40 \mathrm{~mm})$. For the reconstruction of FMT, the in vivo mouse model was discretized into

Table 5 Optical properties of the mouse organs and tissues.

\begin{tabular}{lccccc}
\hline Material & $\begin{array}{c}\text { Muscle } \\
\left(\mathrm{m}^{-1}\right)\end{array}$ & $\begin{array}{c}\text { Lungs } \\
\left(\mathrm{m}^{-1}\right)\end{array}$ & $\begin{array}{c}\text { Heart } \\
\left(\mathrm{m}^{-1}\right)\end{array}$ & $\begin{array}{c}\text { Liver } \\
\left(\mathrm{m}^{-1}\right)\end{array}$ & $\begin{array}{c}\text { Kidneys } \\
\left(\mathrm{m}^{-1}\right)\end{array}$ \\
\hline$\mu_{\mathrm{ax}}$ & 84.5 & 191.82 & 57.4 & 343.7 & 64.4 \\
$\mu_{\mathrm{sx}}^{\prime}$ & 427.3 & 2172.0 & 962.0 & 677.0 & 2248.0 \\
$\mu_{\mathrm{am}}$ & 56.3 & 126.6 & 383.0 & 228.3 & 43.0 \\
$\mu_{\mathrm{sm}}^{\prime}$ & 379.2 & 2124.0 & 905.0 & 648.0 & 2109.0 \\
\hline
\end{tabular}

a volumetric mesh with 5302 nodes and 29,414 tetrahedral elements.

In the same way, we adopted the VSAD and $\mathrm{L}_{2,1}$-norm method to compare with the L1-PAPG method. The parameters were also determined by L-curve method. The results reconstructed by VSAD method, $\mathrm{L}_{2,1}$-norm method, and the L1PAPG method were presented in Fig. 10. Their quantitative comparisons were shown in Table 6. The cross-sections in the $z=6.4 \mathrm{~mm}$ plane and the corresponding CT image were also shown in the second row and third row of Fig. 10. The reconstruction results revealed that both L1-PAPG and $\mathrm{L}_{2,1}$ norm were able to obtain a satisfactory result with the bias of 0.56 and $0.55 \mathrm{~mm}$, whereas the result of VSAD method located a large bias of the fluorescent bead with $0.84 \mathrm{~mm}$. However, the reconstruction time of the L1-PAPG method was $6.84 \mathrm{~s}$, which was faster than $\mathrm{L}_{2,1}$-norm method (7.33 s). This in vivo experiment demonstrates that the L1-PAPG method is efficient and fast for FMT reconstruction. The results implied that the proposed method has potential to practical application.

\section{Conclusion and Discussion}

In this study, L1-PAPG method based on primal accelerated gradient descent and L1RP projection for FMT problem has been proposed. As we all know, FMT is an ill-posed and illconditioned problem. To improve the reconstruction results, many regularized models are utilized to solve the problem, such as the interior-point method, the CG_L2 method, the IS_L1 method, L1-PCLS method, and StOMP. However, the robust of these methods need to be further improved. In this paper, the L1-PAPG was proposed to reconstruct fluorescent sources in the biological tissue. To assess the performance, simulation experiments, mouse phantom experiments, and in vivo small mouse experiments were designed. The results showed that the accuracy of the proposed method was better than VSAD but not as good as $\mathrm{L}_{2,1}$-norm. However, the PE gap between the L1-PAPG and $\mathrm{L}_{2,1}$-norm method was small, and the proposed method has its own advantages. First, it does not need the prior information of structure sparsity but purely based on the sparsity. Thus, the proposed method is likely to be more universal and feasible for applying FMT in different scenarios. For example, it is more suitable for small tumor reconstruction, because small tumors have strong characteristic of sparsity. Second, since the proposed method only utilizes the sparsity information, the computational complex is less than the $\mathrm{L}_{2,1}$-norm method. Thus, its reconstruction speed is faster. This was proved by the experiments in our manuscript. Therefore, the $\mathrm{L}_{2,1}$-norm method is more suitable for the cases that require

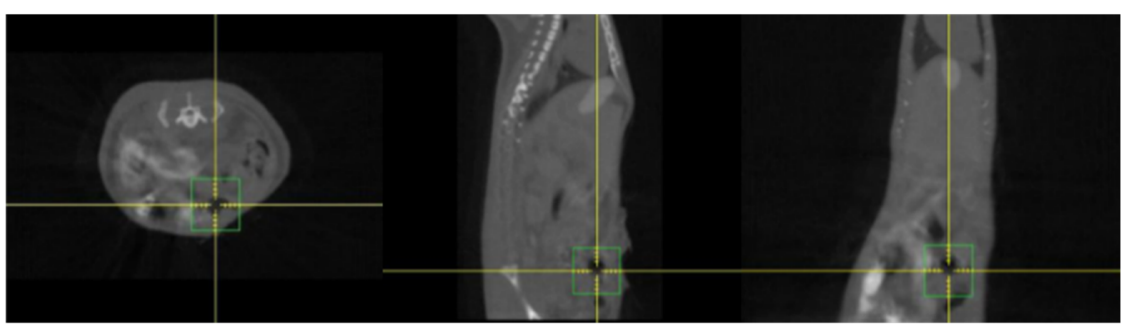

(a)

(b)

(c)

Fig. 9 Micro-CT images of the small mouse. (a) Transverse view, (b) sagittal view, and (c) coronal view. 


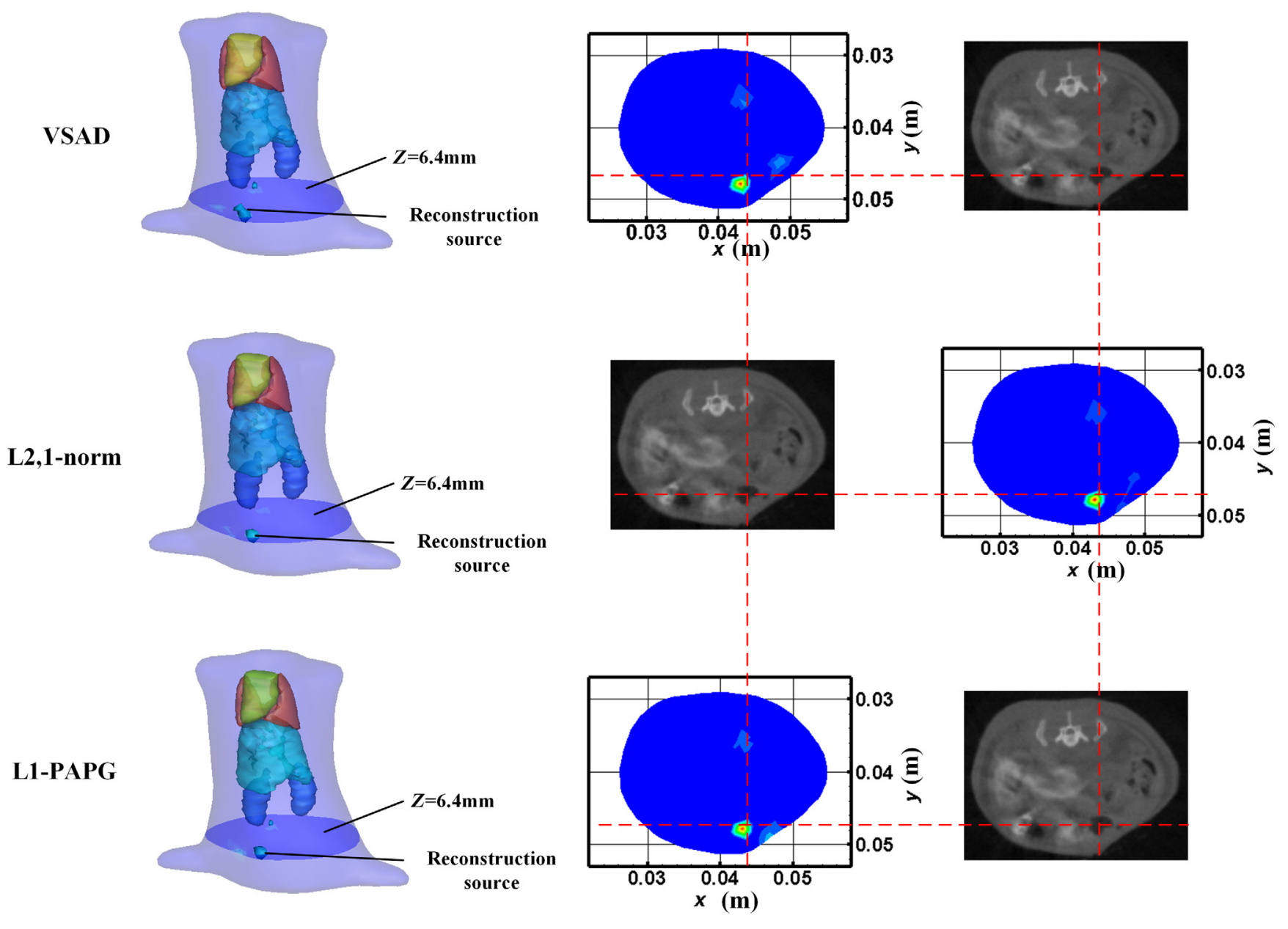

Fig. 10 The reconstruction results of different methods.

Table 6 Quantitative analysis of the in vivo mouse experiment.

\begin{tabular}{lccc}
\hline & & & \\
& VSAD & $\mathrm{L}_{2,1}$-norm & L1-PAPG \\
\hline PE $(\mathrm{mm})$ & 0.84 & 0.55 & 0.56 \\
SBR & 58.52 & 65.65 & 60.57 \\
Time $(\mathrm{s})$ & 14.66 & 7.33 & 6.84 \\
\hline
\end{tabular}

higher precision, and the structural prior information is easy to obtain. The proposed method is more suitable for the cases that require higher speed, and structural prior is difficult to obtain. The experiment results also demonstrated that the L1-PAPG method was robust to noise and had great potential on the practical application of FMT.

In conclusion, the L1-PAPG method is a robust and efficient reconstruction strategy for FMT. Although the L1-PAPG can achieve promising results in FMT, there are still some challenging problems for FMT reconstruction, such as the morphological reconstruction of the tumor, which has a great effect on treatment in the field of oncology. The future work may focus on the tumor boundary determination and morphological reconstruction of the tumor.

\section{Disclosures}

The authors declare no conflict of interest. The animal experiment was conducted under approved research protocols of the Institutional Animal Care and Use Committee, Chinese Academy of Sciences.

\section{Acknowledgments}

This work is supported by the National Key Research and Development Program of China under Grant Nos. 2017YFA0700401 and 2017YFA0205200, the National Natural Science Foundation of China under Grant Nos. 81571836, 61601019, 81527805, and 61671449, the Beijing Natural Science Foundation under Grant No. 7164270, the Fundamental Research Funds for Central Universities under Grant Nos. 2017RC023 and 2017RC025, the International Innovation Team of CAS under Grant No. 20140491524, Beijing Municipal Science \& Technology Commission under Grant No. Z161100002616022, the General Financial Grant from the China Postdoctoral Science Foundation under Grant No. 2017M620952, and the 111 Project under Grant No. B13003.

\section{References}

1. V. Ntziachristos, "Fluorescence molecular imaging," Апnи. Rev. Biomed. Eng. 8, 1-33 (2006). 
2. A. Ale et al., "FMT-XCT: in vivo animal studies with hybrid fluorescence molecular tomography-X-ray computed tomography," Nat. Methods 9(6), 615-620 (2012).

3. A. Cong and G. Wang, "A finite-element-based reconstruction method for 3D fluorescence tomography," Opt. Express 13(24), 9847-9857 (2005).

4. F. Leblond et al., "Toward whole-body optical imaging of rats using single-photon counting fluorescence tomography," Opt. Lett. 36(19), 3723-3725 (2011).

5. M. L. James and S. S. Gambhir, "A molecular imaging primer: modalities, imaging agents, and applications," Physiol. Rev. 92(2), 897-965 (2012).

6. G. Zhang et al., "Acceleration of dynamic fluorescence molecular tomography with principal component analysis," Biomed. Opt. Express 6(6), 2036-2055 (2015)

7. Q. Zhao et al., "A handheld fluorescence molecular tomography system for intraoperative optical imaging of tumor margins," Med. Phys. 38(11), 5873-5878 (2011).

8. M. A. Whitney et al., "Fluorescent peptides highlight peripheral nerves during surgery in mice," Nat. Biotechnol. 29(4), 352-356 (2011).

9. S. R. Mudd et al., "Molecular imaging in oncology drug development," Drug Discovery Today 22(1), 140-147 (2017).

10. C. Chi et al., "Intraoperative imaging-guided cancer surgery: from current fluorescence molecular imaging methods to future multi-modality imaging technology," Theranostics 4(11), 1072-1084 (2014).

11. V. Ntziachristos, "Going deeper than microscopy: the optical imaging frontier in biology," Nat. Methods 7(8), 603-614 (2010).

12. Y. An et al., "Compactly supported radial basis function-based meshless method for photon propagation model of fluorescence molecular tomography," IEEE Trans. Med. Imaging 36(2), 366-373 (2017).

13. Y. An et al., "A novel region reconstruction method for fluorescence molecular tomography," IEEE Trans. Biomed. Eng. 62(7), 1818-1826 (2015).

14. S. Jiang et al., "Novel 1 2, 1-norm optimization method for fluorescence molecular tomography reconstruction," Biomed. Opt. Express 7(6), 2342-2359 (2016).

15. J. Ye et al., "Reconstruction of fluorescence molecular tomography via a nonmonotone spectral projected gradient pursuit method," J. Biomed. Opt. 19(12), 126013 (2014).

16. N. Deliolanis et al., "Free-space fluorescence molecular tomography utilizing 360 degrees geometry projections," Opt. Lett. 32(4), 382-384 (2007).

17. W. Zhu et al., "Iterative total least-squares image reconstruction algorithm for optical tomography by the conjugate gradient method," J. Opt. Soc. Am. A 14(4), 799-807 (1997).

18. W. Bangerth and A. Joshi, "Adaptive finite element methods for the solution of inverse problems in optical tomography," Inverse Probl. 24(3), 034011 (2008).

19. F. H. Tian et al., "Enhanced functional brain imaging by using adaptive filtering and a depth compensation algorithm in diffuse optical tomography," IEEE Trans. Med. Imaging 30(6), 1239-1251 (2011).

20. P. Mohajerani and V. Ntziachristos, "An inversion scheme for hybrid fluorescence molecular tomography using a fuzzy inference system," IEEE Trans. Med. Imaging 35(2), 381-390 (2016).

21. D. L. Donoho, "Compressed sensing," IEEE Trans. Inf. Theory 52(4), 1289-1306 (2006).

22. D. Han et al., "A fast reconstruction algorithm for fluorescence molecular tomography with sparsity regularization," Opt. Express 18(8), 86308646 (2010).

23. J. W. Shi et al., "Efficient L1 regularization-based reconstruction for fluorescent molecular tomography using restarted nonlinear conjugate gradient," Opt. Lett. 38(18), 3696-3699 (2013).

24. V. C. Kavuri et al., "Sparsity enhanced spatial resolution and depth localization in diffuse optical tomography," Biomed. Opt. Express 3(5), 943-957 (2012).

25. J. Ye et al., "Sparse reconstruction of fluorescence molecular tomography using variable splitting and alternating direction scheme," Mol. Imaging Biol. 20(1), 37-46 (2018).

26. D. Zhu and C. Li, "Nonconvex regularizations in fluorescence molecular tomography for sparsity enhancement," Phys. Med. Biol. 59(12), 2901-2912 (2014).

27. N. Ducros et al., "Multiple-view fluorescence optical tomography reconstruction using compression of experimental data," Opt. Lett. 36(8), 1377-1379 (2011).
28. P. Mohajerani and V. Ntziachristos, "Compression of born ratio for fluorescence molecular tomography/x-ray computed tomography hybrid imaging: methodology and in vivo validation," Opt. Lett. 38(13), 23242326 (2013)

29. C. Chen et al., "Diffuse optical tomography enhanced by clustered sparsity for functional brain imaging," IEEE Trans. Med. Imaging 33(12), 2323-2331 (2014).

30. T. K. Pong et al., "Trace norm regularization: reformulations, algorithms, and multi-task learning," SIAM J. Optim. 20(6), 3465-3489 (2010).

31. J. Liu and J. Ye, "Efficient Euclidean projections in linear time," Int. Conf. on Machine Learning, pp. 657-664 (2009).

32. J. H. Lee, A. Joshi, and E. M. Sevick-Muraca, "Fully adaptive finite element based tomography using tetrahedral dual-meshing for fluorescence enhanced optical imaging in tissue," Opt. Express 15(11), 69556975 (2007).

33. W. Cong et al., "Practical reconstruction method for bioluminescence tomography," Opt. Express 13(18), 6756-6771 (2005).

34. A. Joshi, W. Bangerth, and E. M. Sevick-Muraca, "Adaptive finite element based tomography for fluorescence optical imaging in tissue," Opt. Express 12(22), 5402-5417 (2004).

35. J. Z. Huang, T. Zhang, and D. Metaxas, "Learning with structured sparsity," J Mach. Learn. Res. 12, 3371-3412 (2011).

36. R. G. Baraniuk et al., "Model-based compressive sensing," IEEE Trans. Inf. Theory 56(4), 1982-2001 (2010).

37. V. Cevher et al., "Recovery of clustered sparse signals from compressive measurements," in Int. Conf. on Sampling Theory and Applications, Vol. 78, No. 7, pp. 599-606 (2009).

38. P. C. Hansen et al., "An adaptive pruning algorithm for the discrete L-curve criterion 动, J. Comput. Appl. Math. 198(2), 483-492 (2007).

39. M. Li et al., "Reconstruction of fluorescence molecular tomography using a neighborhood regularization," IEEE Trans. Biomed. Eng. 59(7), 1799-1803 (2012).

40. M. Chen et al., "Automatic selection of regularization parameters for dynamic fluorescence molecular tomography: a comparison of L-curve and U-curve methods," Biomed. Opt. Express 7(12), 5021 (2016).

41. P. C. Hansen, "Regularization tools: a MATLAB package for analysis and solution of discrete ill-posed problems," Numer. Algorithms $\mathbf{6}(1)$, 1-35 (1994).

42. S. L. Jacques, "Optical properties of biological tissues: a review," Phys. Med. Biol. 58(11), R37-R61 (2013).

43. W. Ping et al., "Bioluminescence tomography by an iterative reweighted (1)2 norm optimization," IEEE Trans. Biomed. Eng. 61(1), 189-196 (2014).

44. C. Qin, S. Zhu, and J. Tian, "New optical molecular imaging systems," Curr. Pharm. Biotechnol. 11(6), 620-627 (2010).

45. S. Zhu et al., "Cone beam micro-CT system for small animal imaging and performance evaluation," Int. J. Biomed. Imaging 2009, 1-9 (2009).

46. X. L. Chen et al., "3D reconstruction of light flux distribution on arbitrary surfaces from 2D multi-photographic images," Opt. Express 18(19), 19876-19893 (2010).

47. F. Gao et al., "A linear, featured-data scheme for image reconstruction in time-domain fluorescence molecular tomography," Opt. Express 14(16), 7109-7124 (2006).

48. G. R. Yan et al., "Fast cone-beam CT image reconstruction using GPU hardware," J. X-Ray Sci. Technol. 16(4), 225-234 (2008).

49. G. Alexandrakis, F. R. Rannou, and A. F. Chatziioannou, "Tomographic bioluminescence imaging by use of a combined optical-PET (OPET) system: a computer simulation feasibility study," Phys. Med. Biol. 50(17), 4225-4241 (2005).

Yuhao Liu just received his MS degree in biomedical engineering from Beijing Jiaotong University in 2018, and he received his BS degree in electronic information science and technology from Shandong University of Science and Technology in 2015. His main research interests include fluorescence molecular imaging.

Shixin Jiang is currently a PhD student with Beijing Jiaotong University, School of Computer and Information Technology. He received his BS degree in biomedical engineering from Beijing Jiaotong University in 2014. His main research interests include multimodality molecular imaging and medical image processing. 
Jie Liu is a professor in the School of Computer and Information, Beijing Jiaotong University. His research interests include medical image processing and molecular imaging.

Yu An is a postdoctor in CAS Key laboratory of Molecular Imaging, Institute of Automation, Chinese Academy of Sciences. His main research interests include multimodality molecular imaging.

Guanglei Zhang is currently an associate professor in Beijing Advanced Innovation Center for Biomedical Engineering, School of Biological Science and Medical Engineering, Beihang University. His main research interests include fluorescence molecular tomography.

Yuan Gao is currently a PhD student in CAS Key laboratory of Molecular Imaging, Institute of Automation, Chinese Academy of
Sciences. His main research interests include bioluminescence tomography.

Kun Wang is an associate professor in the CAS Key Laboratory of Molecular Imaging, Institute of Automation, Chinese Academy of Sciences. His main research interests include multimodality molecular imaging.

Jie Tian is a professor in the CAS Key Laboratory of Molecular Imaging, Institute of Automation, Chinese Academy of Sciences. His research interests covered in medical image processing and analysis, molecular imaging, pattern recognition. $\mathrm{He}$ is the fellow of SPIE, IEEE, IAMBE, AIMBE, and IAPR. 